\title{
Value of amniotic fluid homocysteine assay in prenatal diagnosis of combined methylmalonic acidemia and homocystinuria, cobalamin C type
}

Ting Chen ${ }^{1,2+}$, Lili Liang ${ }^{1,2+}$, Huiwen Zhang ${ }^{1,2}$, Jun Ye ${ }^{1,2}$, Wenjuan Qiu ${ }^{1,2}$, Bing Xiao ${ }^{1,2}$, Hong Zhu ${ }^{2}$, Lei Wang ${ }^{2}$, Feng $\mathrm{Xu}^{1,2}$, Zhuwen Gong ${ }^{1,2}$, Xuefan $\mathrm{Gu}^{1,2}$ and Lianshu Han ${ }^{1,2^{*}}$

\begin{abstract}
Background: Combined methylmalonic acidemia and homocystinuria, cobalamin C type (cblC defect) is the most common inborn error of cobalamin metabolism, and different approaches have been applied to its prenatal diagnosis. To evaluate the reliability of biochemical method for the prenatal diagnosis of cblC defect, we conducted a retrospective study of our 10-year experience at a single center.
\end{abstract}

Methods: 248 pregnancies whose probands were diagnosed as cblC defect were referred to our center for prenatal diagnosis from January 2010 to December 2019. Prenatal data of Hcy levels determined by enzymatic cycling assay, acylcarnitine analysis using liquid chromatography tandem mass spectrometry, organic acid analysis using gas chromatography mass spectrometry, and genetic analysis by direct sequencing of 248 at-risk fetuses were retrospectively reviewed.

Results: For 2.0 and $16.0 \mu \mathrm{mol} / \mathrm{L}$ levels of Hcy AF samples, the relative errors were $-2.5 \%$ and $2.8 \%$, respectively. The respective measurement uncertainties were $13.07 \%$ and 14.20\%. For the 248 at-risk fetuses, 63 fetuses were affected and 185 fetuses were unaffected. Hcy level of 13.20 (6.62-43.30) $\mu \mathrm{mol} / \mathrm{L}$ in 63 affected fetuses was significantly higher than that in 185 unaffected fetuses of $2.70(0.00-5.80) \mu \mathrm{mol} / \mathrm{L}$, and there was no overlap between the affected and unaffected groups. The diagnostic sensitivity and specificity of Hcy were $100 \%$ and $92.05 \%$, respectively. The positive and negative predictive values of the combination of Hcy, propionylcarnitine (C3), ratio of C3 to acetylcarnitine (C2; C3/C2), methylmalonic acid (MMA), and methylcitric acid (MCA) were both 100\%. Sixteen fetuses displayed inconclusive genetic results of MMACHC variants, in which seven fetuses were determined to be affected with elevated levels of Hcy, C3, C3/C2 and MMA, and their levels were 18.50 (6.70-43.30) $\mathrm{mmol} / \mathrm{L}, 8.53(5.02-11.91) \mu \mathrm{mol} / \mathrm{L}, 0.77$ (0.52-0.97), $8.96(6.55-40.32) \mathrm{mmol} / \mathrm{mol} \mathrm{Cr}$, respectively. The remaining nine fetuses were considered unaffected based on a normal amniotic fluid metabolite profile.

\footnotetext{
*Correspondence: hanlianshu@xinhuamed.com.cn

'Ting Chen and Lili Liang have contributed equally to this work

${ }^{1}$ Department of Pediatric Endocrinology and Genetic, Xinhua

Hospital, Shanghai Institute for Pediatric Research, Shanghai Jiao Tong

University School of Medicine, 1665 Kongjiang Road, Yangpu District,

Shanghai 200092, China

Full list of author information is available at the end of the article
}

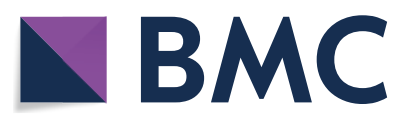

(c) The Author(s) 2021. Open Access This article is licensed under a Creative Commons Attribution 4.0 International License, which permits use, sharing, adaptation, distribution and reproduction in any medium or format, as long as you give appropriate credit to the original author(s) and the source, provide a link to the Creative Commons licence, and indicate if changes were made. The images or other third party material in this article are included in the article's Creative Commons licence, unless indicated otherwise in a credit line to the material. If material is not included in the article's Creative Commons licence and your intended use is not permitted by statutory regulation or exceeds the permitted use, you will need to obtain permission directly from the copyright holder. To view a copy of this licence, visit http://creativecommons.org/licenses/by/4.0/. The Creative Commons Public Domain Dedication waiver (http://creativeco mmons.org/publicdomain/zero/1.0/) applies to the data made available in this article, unless otherwise stated in a credit line to the data. 
Conclusions: Hcy appears to be another characteristic biomarker for the prenatal diagnosis of cblC defect. The combination of Hcy assay with acylcarnitine and organic acid analysis is a fast, sensitive, and reliable prenatal diagnostic biochemical approach. This approach could overcome the challenge of the lack of genetic analysis for families with at-risk cblC defect fetuses.

Keywords: Methylmalonic academia, Homocysteine, Prenatal diagnosis, MMACHC variant

\section{Introduction}

Combined methylmalonic academia and homocystinuria, cobalamin $\mathrm{C}$ type (cblC defect) is the most frequent genetic disorder of cobalamin metabolism [1]. The incidence of cblC defect ranges from 1:46,000 to 1:200,000 in European and American countries [2] and varies hugely from $1: 3,220$ to $1: 21,488$ in China [3-5].The cblC defect is caused by variants in the $M M A C H C$ gene located in chromosome region $1 \mathrm{p} 34.1$ [6]. This defect impairs the conversion of cobalamin to methylcobalamin and adenosylcobalamin, resulting in the accumulation of homocysteine (Hcy) and methylmalonic acid (MMA) [7]. Based on the age of onset, cblC defect has two distinct phenotypes. Patients with early-onset present clinical symptoms that include feeding difficulties, progressive developmental delay, and hypotonia within the first year of life. Patients with late-onset exhibit relatively milder clinical features, such as behavioral disturbances and progressive neurological symptoms, later in life [8]. Despite the early diagnosis and effective treatment, the outcome is not always favorable, especially in early-onset cblC defect [9-11]. Thus, a reliable method for the prenatal diagnosis of cblC defect is needed to inform decisions regarding continuation of pregnancies of cblC defect fetuses.

To some extent, the combination of acylcarnitine analysis (characteristic metabolite biomarkers of propionylcarnitine (C3), the ratio of $\mathrm{C} 3$ to acetylcarnitine (C2; C3/ $\mathrm{C} 2)$ by liquid chromatography tandem mass spectrometry (LC-MS/MS), organic acid analyses of characteristic metabolite biomarkers of MMA, and methylcitric acid (MCA) by gas chromatography mass spectrometry (GCMS) is widely applied to the prenatal diagnosis of cblC defect $[12,13]$. False positive and false negative results might also exist [14]. Thus, identifying another characteristic and sensitive biomarker to enhance the accuracy of metabolite analysis is desirable for the prenatal diagnosis of cblC defect. As cblC defect patients display a marked elevation of the plasma Hcy level $[15,16]$, an elevated Hcy level in amniotic fluid (AF) might serve as a characteristic metabolite biomarker for the prenatal diagnosis of cblC defect. Furthermore, the combination of Hcy assay with acylcarnitine and organic acid analysis in AF could be more reliable and precise for the prenatal diagnosis of cblC defect.
Here, we present our findings with 248 at-risk pregnancies whose probands were diagnosed as cblC defect in the prenatal diagnosis of cblC defect by metabolite analysis of AF supernatants with/without genetic analysis of amniocytes. The aim was to elucidate the value of AF Hcy assay in the prenatal diagnosis of cblC defect.

\section{Methods}

\section{Families and probands}

In this study, 226 families (248 pregnancies) in which the probands were diagnosed as cblC defect were referred to our center for prenatal diagnosis from January 2010 to December 2019. The probands were diagnosed based on the symptoms, metabolite results of elevated blood levels of $\mathrm{C} 3, \mathrm{C} 3 / \mathrm{C} 2$, MMA and MCA, and/or with genetic analysis. Informed consent forms were signed by the parents or legal guardians of the study participants. This study was approved by the Ethics Committee of Xinhua Hospital (approval number XHEC-D-2020-131).

\section{AF sample}

In each case, $30 \mathrm{~mL}$ sample of AF was collected at 16-20 weeks of gestation from the pregnant woman. Of the $30 \mathrm{~mL}, 10 \mathrm{~mL}$ was used for DNA extraction. The cellfree AF supernatant was used for metabolite analysis. For this analysis, $13 \mu \mathrm{L}$ was used for Hcy determination. $2 \mathrm{~mL}$ was used for organic acids analysis. $3 \mu \mathrm{L}$ was used for quantitative acylcarnitine analysis. The remaining $20 \mathrm{~mL}$ of AF was cultured for karyotyping analysis, with the cultured amniocytes also used as a back-up.

\section{Hcy determination of $\mathrm{AF}$}

Thirteen microliters of each cell-free AF sample was centrifuged at $3000 \mathrm{r} / \mathrm{min}$ for $5 \mathrm{~min}$. The level of Hcy in AF was measured by an enzymatic cycling assay using the Hcy assay kit (Beijing Jiuqiang Biotechnology, Beijing, China) and following the manufacturer's protocol using an automatic analyzer (Hitachi, Tokyo, Japan). To interpret the results of Hcy assay in AF, the degree of measurement uncertainty and relative error were investigated. A low level of $2.0 \mu \mathrm{mol} / \mathrm{L}$ and a high level of $16.0 \mu \mathrm{mol} / \mathrm{L}$ of Hcy AF samples were measured. Within-day imprecision was assessed by measuring six replicates of the two Hcy levels of AF samples, and values of co-efficient of variation $\left(C V_{W}\right)$ were calculated. 
Between-day imprecision of Hcy assay was assessed by daily analysis for 6 days of a single lot of the two Hcy levels of AF samples, and $C V_{B}$ were calculated. Method bias was assessed by measuring the two standard Hcy levels of AF samples in every 3 days for 6 times, and $C V_{\text {Bias }}$ were calculated. The inspection quality of Hcy assay was assessed by calculating the measurement uncertainty $\left(U=\sqrt{C V_{W}{ }^{2}+C V_{B}^{2}+C V_{\text {Bias }}^{2}} \times \kappa\right)$ with the coverage factor $\kappa=2$.

\section{Acylcarnitine analysis of AF by LC-MS/MS}

The level of acylcarnitine in each AF samples was analyzed using an API-4000 tandem mass spectrometer (Applied Biosystems, Foster City, CA, USA). Pretreatment of each AF sample and the LC-MS/MS operating conditions were previously reported [17]. Quantitative analysis of acylcarnitine was achieved using the ratio of the averaged ion intensity to that of the corresponding internal standards.

\section{Organic acid analysis by GC-MS in AF}

Organic acids in AF were analyzed using a single quadrupole GCMS-QP 2010 device (Shimadzu, Kyoto, Japan). Pretreatment of AF sample and GC-MS operating conditions were previously reported [17]. Quantitative analysis of each organic acid was achieved using the relative peak area of each Q-ion to that of the corresponding internal standards.

\section{Direct $M M A C H C$ variant screening by Sanger sequencing} Genomic DNA was extracted from the cultured amniocytes using the DNA extraction kit (TIANGEN Biotech, Beijing, China) according to the manufacturer's instruction. The conditions of the PCR reactions and analysis of DNA sequencing were previously described [18]. Nucleotide variations were identified using a reference sequence from Genbank (MMACHC: NM_015506). The novel variants were searched using the Mutalyzer website tool (https://mutalyzer.nl/) and the Human Gene Mutation Database.

\section{Diagnostic criteria}

Biochemical analysis: The reference ranges of Hcy, C3, C3/C2, MMA and MCA were1.10-4.10 $\mu \mathrm{mol} / \mathrm{L}, 0.30$ $4.00 \mu \mathrm{mol} / \mathrm{L}, 0.05-0.25,0.00-1.00 \mathrm{mmol} / \mathrm{mol} \mathrm{Cr}$ and $0.00-0.50 \mathrm{mmol} / \mathrm{mol} \mathrm{Cr}$, respectively. Fetuses with the above metabolites' levels higher than the upper limit of reference ranges were suggested as cblC defect.

Genetic analysis: Fetuses harbored homozygous variants or compound heterozygous variants of $M M A C H C$ were diagnosed as cblC defect.

\section{Statistical analysis}

Data analysis was performed using SPSS 24.0 (IBM, Chicago, Illinois). Data of detected metabolites were not normally distributed. The data are presented as median (range). The Mann-Whitney $U$ test was applied to compare the difference of the levels of the metabolites between the affected and unaffected groups. A $p$ value less than 0.05 was considered statistically significant with a $95 \%$ confidence interval.

\section{Results}

The metabolite and genetic analyses involved 248 at-risk fetuses. The combination of these analyses identified 56 fetuses as affected, with 176 fetuses considered unaffected. Of the remaining 16 fetuses with unavailable causative variants in the probands, based on AF metabolites analysis alone, 7 fetuses were determined to be affected and diagnosed as cblC defect, and 9 fetuses were judged as unaffected.

\section{Biochemical analysis of AF metabolites}

The results of AF metabolites inferred that 63 of the totals of 248 fetuses were affected, with the remaining 185 unaffected (Table 1).

\section{Hcy level in AF}

For the standard levels of $2.0 \mu \mathrm{mol} / \mathrm{L}$ and $16.0 \mu \mathrm{mol} / \mathrm{L}$ of Hcy AF samples, the relative errors were $-2.5 \%$ and $2.8 \%$, respectively. The respective within-day imprecisions were $2.13 \%$ and $1.61 \%$, the respective between-day imprecisions were $4.45 \%$ and $3.90 \%$, and the respective method bias imprecisions were $4.29 \%$ and $5.71 \%$. The measurement uncertainties of the selected two levels ( 2.0 and $16.0 \mu \mathrm{mol} / \mathrm{L}$ ) of Hcy were $13.07 \%$ and $14.20 \%$, respectively (Table 1 ). For the 185 unaffected fetuses, the median (range) level of Hcy was $2.70 \mu \mathrm{mol} / \mathrm{L}(0.00-5.80)$, which was significantly elevated in 63 affected fetuses with a median (range) level of $13.20 \mu \mathrm{mol} / \mathrm{L}(6.62-43.30)$ $(p<0.0001$; Fig. 1). There was no overlap of the Hcy level between the affected and unaffected fetuses. All the individual levels of Hcy in 63 affected fetuses were higher

Table 1 Measurement uncertainties of two levels of Hcy in amniotic fluid

\begin{tabular}{llllll}
\hline $\begin{array}{l}\text { True value of } \\
\text { Hcy }(\mu \mathrm{mol} / \mathrm{L})\end{array}$ & $\begin{array}{l}\text { Measured } \\
\text { median value } \\
\text { of Hcy }(\boldsymbol{\mu m o l} / \mathrm{L})\end{array}$ & $C V_{W}(\%)$ & $C V_{B}(\%)$ & $C V_{\text {Bias }}(\%)$ & $U(\%)$ \\
\hline 2.00 & 1.95 & 2.13 & 4.45 & 4.29 & 13.07 \\
16.00 & 16.45 & 1.61 & 3.90 & 5.71 & 14.20 \\
\hline
\end{tabular}

Hcy homocysteine, $C V_{w}$ within-day imprecision, $C V_{B}$ between-day imprecision, $C V_{\text {Bias }}$ method bias imprecision, $U$ measurement uncertainty 

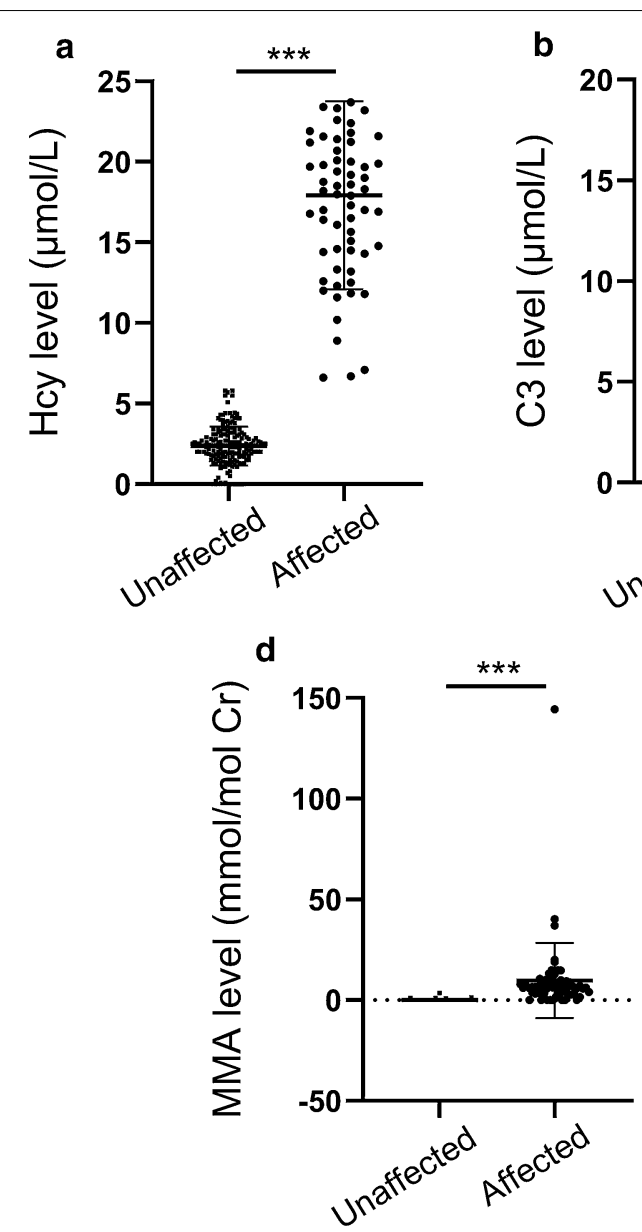
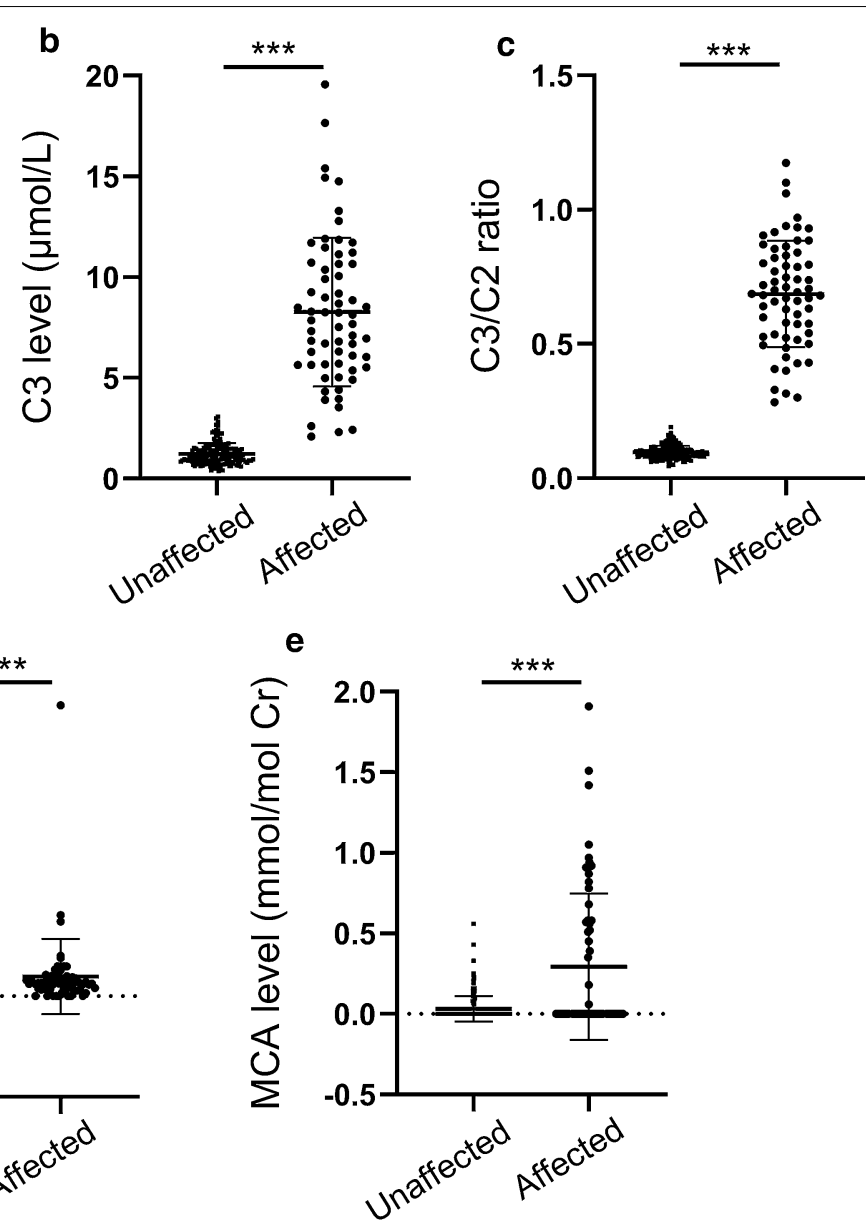

Fig. 1 Scatter-plot showing the distribution of characteristic metabolite levels in affected and unaffected fetuses. a The distribution of Hcy levels between affected and unaffected fetuses; $\boldsymbol{b}$ the distribution of $\mathrm{C} 3$ levels between affected and unaffected fetuses; $\mathbf{c}$ the distribution of $\mathrm{C} 3 / \mathrm{C} 2$ ratios between affected and unaffected fetuses; $\mathbf{d}$ the distribution of MMA levels between affected and unaffected fetuses; $\mathbf{e}$ the distribution of MCA levels between affected and unaffected fetuses. ${ }^{* *}$ Significantly difference $(p<0.001)$ between the affected and unaffected groups

than the defined reference range. Among the 185 unaffected fetuses, the Hcy levels in 15 fetuses were above the upper limit of the reference range.

\section{Acylcarnitine and organic acid levels in AF}

As shown in Fig. 1, the median (range) levels of C3, C3/ C2, MMA, and MCA in the AF of 185 unaffected fetuses were $1.08(0.37-3.07) \mu \mathrm{mol} / \mathrm{L}, 0.09(0.05-0.19), 0.00$ $(0.00-3.65) \mathrm{mmol} / \mathrm{mol} \mathrm{Cr}$, and $0.00(0.00-0.56) \mathrm{mmol} /$ mol $\mathrm{Cr}$, respectively. These levels were notably increased in the 63 affected fetuses with the corresponding metabolite median (range) levels of $8.01(2.09-19.58) \mu \mathrm{mol} / \mathrm{L}$, $0.69(0.28-1.17), 6.22(0.00-144.40) \mathrm{mmol} / \mathrm{mol} \mathrm{Cr}$, and $0.00(0.00-1.91) \mathrm{mmol} / \mathrm{mol} \mathrm{Cr}$, respectively $(p<0.0001)$. Among the 63 affected fetuses, the C3 levels in 7 fetuses, MMA levels in 8 fetuses, and MCA levels in 44 fetuses were within the reference range. Among the 185 unaffected fetuses, the MMA levels in 4 fetuses and the MCA level in one fetus were above the upper limit of the reference range.

\section{Genetic analysis of pathogenic variants in amniocyte DNA}

Among the 248 at-risk fetuses, there were 232 fetuses with clear information concerning pathogenic variants in the probands and parents. For the 232 probands and 56 affected fetuses with clear $M M A C H C$ variants information, 59 cases harbored homozygous variants. The remaining 229 cases harbored compound heterozygous variants. Forty different variants were found. Of these, 9 variants were novel (see Additional file 1). The c.609G > A and c.658_660delAAG variants were the most common variants and accounted for $61.15 \%$ of disease alleles.

A total of 16 fetuses displayed inconclusive genetic results. Of these, 12 fetuses had only one causative mutation in the probands and there was a lack of available genetic information in the probands in 4 fetuses. 
Comparison of metabolite results and genetic results Among the 232 fetuses with clear $M M A C H C$ variants information in the probands and parents, genetic and metabolite results in 169 fetuses were completely consistent. Twelve fetuses were affected and the remaining 157 were unaffected. For 63 fetuses, the metabolite results of Hcy, C3, C3/C2, MMA, and MCA were incompletely consistent with the genetic results (Table 2). Of these 63 fetuses, 44 were affected and 19 were unaffected. For the 44 affected fetuses, compared with the genetic results, inconsistent findings were evident for $\mathrm{C} 3$ of 3 fetuses, MMA of one fetus, MCA of 29 fetuses, and C3 and MCA of 4 fetuses. For the remaining 7 fetuses, the MMA and MCA results were inconsistent. For the 19 unaffected fetuses, inconsistencies with the genetic results were evident for the Hcy of 14 fetuses, MMA of 4 fetuses, and MCA of one fetus.

Among the 16 fetuses with inconclusive genetic results, 7 were inferred to be affected. Of these 7 fetuses, the levels of all metabolites were elevated in 3 fetuses. In the remaining 4 fetuses, the levels of $\mathrm{Hcy}, \mathrm{C} 3, \mathrm{C} 3 / \mathrm{C} 2$, and MMA were elevated, while the MCA level was not. For the other 9 unaffected fetuses, the levels of all the metabolites were normal in 8 , with only the Hcy level being increased in the remaining fetus. These 9 fetuses showed a normal phenotype at postnatal follow-up (see Additional file 2).

For the 232 fetuses with information of the pathogenic variants in the probands and parents, comparison of the metabolite results with genetic results revealed the sensitivity and specificity of Hcy were $100 \%$ and $92.05 \%$, respectively. The positive predictive values of $\mathrm{Hcy}, \mathrm{C} 3$, C3/C2, MMA, and MCA were $80 \%, 100 \%, 100 \%, 92.31 \%$, and $94.12 \%$, respectively. The respective negative predictive values were $100 \%, 96.17 \%, 100 \%, 95.56 \%$, and $81.39 \%$. The positive and negative predictive values of the combination of those metabolites were both $100 \%$.

\section{Discussion}

The cblC defect is the most common subtype of vitamin $\mathrm{B}_{12}$ metabolism [19]. Even with rapid diagnosis and effective treatment, the long-term outcome remains unsatisfactory, especially in patients with early onset, because of severe neurological sequelae [11]. The families of these patients suffer heavy economic burdens. Thus, prenatal diagnosis can provide important information in the decision about the pregnancy involving a fetus with the cblC defect, which could further reduce the social and family pressures from this disease.

For the prenatal diagnosis of cblC defect, metabolites can be measured and monitored in AF [20]. In a notable example, Ji et al. [14] reported that metabolite analysis of acylcarnitines by LC-MS/MS and organic acids by
GC-MS in AF could serve as rapid and reliable methods for the prenatal diagnosis of methylmalonic acidemia. However, their data of C3, C3/C2, MMA, and MCA sensitivity $(95.1 \%, 100 \%, 100 \%$, and $82.9 \%$, respectively) and specificity (98.7\%, 99.3\%, 97.4\%, and 96.7\%, respectively) indicated the possibility of false positive results for the analyses of acylcarnitines and organic acids. Therefore, we aimed to find another effective biomarker to enhance the accuracy of metabolite analysis for prenatal diagnosis of the cblC defect.

Plasma total Hcy is recommended as an biomarker in the guideline for the diagnosis of cblC defect patients [15]. The Hcy assay of AF was performed in 9 at-risk fetuses for the prenatal diagnosis of cblC defect [21]. However, published reports concerning Hcy assay in $\mathrm{AF}$ for the prenatal diagnosis of cblC defect were all case reports or small series [21, 22]. Thus, it has been difficult to verify the reliability of the Hcy assay in the prenatal diagnosis of cblC defect. In this context, we retrospectively reviewed 248 at-risk fetuses with prenatal diagnostic data collected over a 10 -year period to analyze the value of Hcy in the prenatal diagnosis of cblC defect. We first assessed the imprecisions of Hcy assay, the $C V_{W}, C V_{B}$ and $C V_{\text {Bias }}$ values were all less than $10 \%$ [23] and proved that Hcy assay in AF was stable. Moreover, to interpret the results of Hcy assay in AF, the relative errors of the selected two levels $(2.0$ and $16.0 \mu \mathrm{mol} / \mathrm{L})$ of Hcy were $-2.5 \%$ and $2.8 \%$, respectively. The respective measurement uncertainties were $13.07 \%$ and $14.2 \%$. Besides, we observed no overlap of the Hcy level between the affected and unaffected fetuses. By contrast, C3/C2 displayed no overlap, while the levels of C3, MMA, and MCA all overlapped between the affected and unaffected fetuses.

Among the 232 fetuses with information concerning pathogenic variants in the probands and parents, results of the Hcy, C3, C3/C2, MMA, and MCA metabolites showed discrepancies in 63 fetuses. Consequently, a biochemical prenatal diagnosis of cblC defect in these fetuses could be uncertain based on any one of the metabolites. Nevertheless, we noted that Hcy levels and $\mathrm{C} 3 / \mathrm{C} 2$ were consistent with genetic results in all affected fetuses, while in all unaffected fetuses, $\mathrm{C} 3$ and $\mathrm{C} 3 / \mathrm{C} 2$ levels were completely consistent with the genetic results. Thus, by taking advantage of the $100 \%$ sensitivity, Hcy could help to decrease the false negative rate and obtain a more accurate biochemical prenatal diagnosis in the affected fetuses. However, in contrast with $\mathrm{C} 3, \mathrm{C} 3 / \mathrm{C} 2$, MMA, and MCA, Hcy showed the lowest specificity of $92.05 \%$, which might be associated with the selection of the reference range. Generally, the setting of the reference range is calculated by taking twice the standard deviation from the mean or considering certain percentiles of the normal 
Table 2 Prenatal data of 63 fetuses with one or more metabolites results inconsistent with genetic results

\begin{tabular}{|c|c|c|c|c|c|c|c|}
\hline \multirow[t]{2}{*}{ No. } & \multicolumn{2}{|c|}{ Variants of fetus (NM_015506) } & \multicolumn{5}{|l|}{ Metabolites } \\
\hline & Allele 1 & Allele 2 & $\mathrm{Hcy}(\mu \mathrm{mol} / \mathrm{L})$ & $\mathrm{C} 3(\mu \mathrm{mol} / \mathrm{L})$ & $\mathrm{C} 3 / \mathrm{C} 2$ & $\mathrm{MMA}(\mathrm{mmol} / \mathrm{mol} \mathrm{Cr})$ & $\mathrm{MCA}(\mathrm{mmol} / \mathrm{mol} \mathrm{Cr})$ \\
\hline 1 & C. $80 A>G$ & c.658_660delAAG & 13.32 & 2.42 & 0.30 & 1.55 & 0.51 \\
\hline 2 & c.568insT & c. $467 \mathrm{G}>\mathrm{A}$ & 18.30 & 5.37 & 0.85 & 0 & 0.92 \\
\hline 3 & C. $80 A>G$ & $C .481 C>T$ & 22.41 & 4.41 & 0.66 & 8.49 & 0 \\
\hline 4 & $C .481 C>T$ & Exon1 deletion & 18.76 & 8.15 & 0.75 & 6.44 & 0 \\
\hline 5 & $\mathrm{C} .80 \mathrm{~A}>\mathrm{G}$ & c.609G $>$ A & 14.60 & 3.96 & 0.43 & 14.75 & 0.97 \\
\hline 6 & c.609G $>$ A & c.656_658delAAG & 13.20 & 10.06 & 0.74 & 5.58 & 0.45 \\
\hline 7 & c.394C>T & c.609G $>A$ & 12.50 & 6.95 & 0.63 & 0 & 0 \\
\hline 8 & c.609G $>A$ & c.656_658delAAG & 16.10 & 6.31 & 0.61 & 0 & 0.55 \\
\hline 9 & c. $482 \mathrm{G}>\mathrm{A}$ & c.609G $>A$ & 7.10 & 6.71 & 0.54 & 0 & 0.06 \\
\hline 10 & C. $80 A>G$ & c.609G $>A$ & 16.90 & 11.15 & 0.58 & 6.57 & 0 \\
\hline 11 & Exon1 deletion & C.599G $>A$ & 28.90 & 14.76 & 1.17 & 144.40 & 0 \\
\hline 12 & $\mathrm{C} .80 \mathrm{~A}>\mathrm{G}$ & c.658_660delAAG & 15.10 & 2.30 & 0.33 & 3.64 & 0 \\
\hline 13 & c.455_457delCCC & c.658_660delAAG & 17.00 & 11.73 & 0.63 & 0 & 0 \\
\hline 14 & c.609G $>A$ & c.445_446delTG & 21.40 & 9.19 & 0.83 & 13.31 & 0 \\
\hline 15 & $c .428 \mathrm{C}>\mathrm{T}$ & c.658_660delAAG & 12.60 & 6.04 & 0.53 & 9.64 & 0 \\
\hline 16 & C. $80 A>G$ & c.609G $>A$ & 23.70 & 7.33 & 0.67 & 5.88 & 0 \\
\hline 17 & c. $80 A>G$ & c. $609 \mathrm{G}>\mathrm{A}$ & 26.60 & 8.69 & 0.82 & 3.35 & 0 \\
\hline 18 & c.394C >T & c.445_446delTG & 27.80 & 8.24 & 0.79 & 9.35 & 0 \\
\hline 19 & c.445_446delTG & c. $609 \mathrm{G}>\mathrm{A}$ & 16.50 & 15.41 & 0.93 & 1.79 & 0 \\
\hline 20 & c.609G $>$ A & c.658_660delAAG & 12.00 & 5.67 & 0.41 & 7.53 & 0 \\
\hline 21 & c.57_58insT & c. $609 \mathrm{G}>\mathrm{A}$ & 18.60 & 11.85 & 0.94 & 6.13 & 0 \\
\hline 22 & c. $609 \mathrm{G}>\mathrm{A}$ & $\begin{array}{l}\text { Carry the same paternal } \\
\text { allele as the proband }\end{array}$ & 20.10 & 14.95 & 0.92 & 6.13 & 0 \\
\hline 23 & $c .217 C>T$ & c. $609 \mathrm{G}>\mathrm{A}$ & 14.30 & 3.90 & 0.50 & 6.24 & 0 \\
\hline 24 & c.609G $>$ A & c.626_627delTG & 14.40 & 12.79 & 0.70 & 7.28 & 0 \\
\hline 25 & c.445_446delTG & c.609G $>A$ & 23.40 & 10.73 & 0.90 & 12.96 & 0 \\
\hline 26 & c.609G $>A$ & c.658_660delAGA & 17.00 & 10.67 & 0.74 & 7.78 & 0 \\
\hline 27 & c.394C>T & c.656_658delAGA & 10.20 & 6.69 & 0.40 & 0 & 0 \\
\hline 28 & c.609G $>$ A & c.658_660delAGA & 17.30 & 7.53 & 0.52 & 6.82 & 0 \\
\hline 29 & C. $80 A>G$ & c.609G $>A$ & 21.80 & 6.11 & 0.49 & 7.09 & 0 \\
\hline 30 & c.567dupT & c.99delA & 19.70 & 19.58 & 0.89 & 6.04 & 0 \\
\hline 31 & c.609G $>$ A & c.658_660delAAG & 19.80 & 10.38 & 0.58 & 3.85 & 0 \\
\hline 32 & c. $80 A>G$ & c.609G $>A$ & 11.80 & 5.70 & 0.32 & 0 & 0 \\
\hline 33 & c.567dupT & c.609G $>A$ & 21.20 & 11.71 & 0.64 & 10.64 & 0 \\
\hline 34 & $c .482 \mathrm{G}>\mathrm{A}$ & c. $482 \mathrm{G}>\mathrm{A}$ & 6.62 & 2.61 & 0.43 & 5.95 & 0.39 \\
\hline 35 & c.445_446delTG & c. $609 \mathrm{G}>\mathrm{A}$ & 19.19 & 3.54 & 0.68 & 3.2 & 1.51 \\
\hline 36 & c. $482 \mathrm{G}>\mathrm{A}$ & c. $482 \mathrm{G}>\mathrm{A}$ & 8.90 & 2.09 & 0.28 & 4.01 & 0 \\
\hline 37 & c.609G $>A$ & c.609G $>A$ & 20.00 & 17.65 & 0.79 & 0 & 0 \\
\hline 38 & c.609G $>$ A & c.609G $>$ A & 19.00 & 9.91 & 0.60 & 5.71 & 0 \\
\hline 39 & c.609G $>$ A & c.658_660delAAG & 22.60 & 6.81 & 0.69 & 2.37 & 0 \\
\hline 40 & c.609G $>$ A & c.609G $>$ A & 16.40 & 7.87 & 0.80 & 5.07 & 0 \\
\hline 41 & c.609G $>A$ & c.609G $>A$ & 19.67 & 9.25 & 0.80 & 14.95 & 0 \\
\hline 42 & c. $609 \mathrm{G}>\mathrm{A}$ & c.609G $>A$ & 17.90 & 6.85 & 0.69 & 37.16 & 0 \\
\hline 43 & c.609G $>A$ & c.658_660delAAG & 21.60 & 11.48 & 0.87 & 6.22 & 0 \\
\hline 44 & c.609G $>$ A & c.658_660delAAG & 20.70 & 13.30 & 0.86 & 6.65 & 0 \\
\hline 45 & $c .217 C>T$ & - & 4.10 & 0.72 & 0.10 & 0 & 0 \\
\hline 46 & c.658_660delAAG & - & 4.40 & 1.46 & 0.15 & 0 & 0.16 \\
\hline 47 & c.658_660delAAG & - & 4.37 & 0.40 & 0.09 & 0.91 & 0.08 \\
\hline
\end{tabular}


Table 2 (continued)

\begin{tabular}{|c|c|c|c|c|c|c|c|}
\hline \multirow[t]{2}{*}{ No. } & \multicolumn{2}{|c|}{ Variants of fetus (NM_015506) } & \multicolumn{5}{|l|}{ Metabolites } \\
\hline & Allele 1 & Allele 2 & Hcy $(\mu \mathrm{mol} / \mathrm{L})$ & $\mathrm{C} 3(\mu \mathrm{mol} / \mathrm{L})$ & $\mathrm{C} 3 / \mathrm{C} 2$ & MMA $(\mathrm{mmol} / \mathrm{mol} \mathrm{Cr})$ & $\mathrm{MCA}(\mathrm{mmol} / \mathrm{mol} \mathrm{Cr})$ \\
\hline 48 & c. $609 \mathrm{G}>\mathrm{A}$ & - & 4.42 & 1.70 & 0.13 & 0 & 0 \\
\hline 49 & c.656_658delAGA & - & 5.70 & 1.05 & 0.09 & 0 & 0.2 \\
\hline 50 & c.658_660delAAG & - & 5.80 & 0.73 & 0.07 & 0 & 0 \\
\hline 51 & c.658_660delAAG & - & 4.30 & 0.52 & 0.08 & 0 & 0 \\
\hline 52 & - & - & 5.50 & 1.47 & 0.09 & 0 & 0 \\
\hline 53 & c. $80 A>G$ & - & 5.50 & 0.97 & 0.16 & 0 & 0 \\
\hline 54 & c. $609 \mathrm{G}>\mathrm{A}$ & - & 5.10 & 2.67 & 0.13 & 0 & 0 \\
\hline 55 & - & - & 4.30 & 1.32 & 0.14 & 0 & 0 \\
\hline 56 & - & - & 4.10 & 3.07 & 0.16 & 0 & 0 \\
\hline 57 & c.609G $>A$ & - & 4.20 & 2.99 & 0.08 & 0 & 0 \\
\hline 58 & - & - & 5.80 & 1.15 & 0.11 & 0 & 0 \\
\hline 59 & - & - & 1.55 & 0.55 & 0.08 & 1.44 & 0 \\
\hline 60 & - & - & 2.60 & 0.60 & 0.11 & 1.06 & 0 \\
\hline 61 & c. $609 \mathrm{G}>\mathrm{A}$ & - & 2.80 & 2.62 & 0.11 & 1.16 & 0 \\
\hline 62 & c.658_660delAAG & - & 2.20 & 0.97 & 0.05 & 3.65 & 0 \\
\hline \multirow[t]{2}{*}{63} & c. $609 \mathrm{G}>\mathrm{A}$ & - & 2.00 & 1.02 & 0.09 & 0 & 0.56 \\
\hline & \multicolumn{2}{|l|}{ Reference range } & $1.10-4.10$ & $0.30-4.00$ & $0.05-0.25$ & $0.00-1.00$ & $0.00-0.50$ \\
\hline
\end{tabular}

Bold parts, metabolite results are inconsistent with the genetic results

Hcy homocysteine, C3 propionylcarnitine, $C 2$ acetylcarnitine, MMA methylmalonic acid, MCA methylcitric acid

population [24].And the reference ranges of biomarkers for clinical diagnosis are commonly set as the 5 th to 95 th percentile of the normal population [25]. Thus, in our study, the reference range of Hcy was determined to be the 5th to 95th percentile of the Hcy values of 109 fetuses who were not at-risk for cblC defect. However, a previous report also suggested that a reference range based exclusively on normal population might result in many false positive results. Thus, the reference range might need adjustment in light of the overlap between the normal and disorder populations [26]. Therefore, depending on the increasing data of AF Hcy and the prenatal diagnosis of cblC defect, the reference range of prenatal Hcy level needs to be adjusted in the future to improve the low specificity of Hcy for the prenatal diagnosis of cblC defect. It is also worth noting that a total of 3 affected fetuses harboring the homozygous or heterozygous variant of c.482G > A displayed lower levels of Hcy (ranging from 6.62 to $8.9 \mu \mathrm{mol} / \mathrm{L}$ ) compared to the other affected fetuses. The connection between the c. $482 \mathrm{G}>\mathrm{A}$ variant and Hcy level warrants a further investigation.

Genetic analysis is generally recognized as the gold standard for prenatal diagnosis [27, 28]. However, despite its accuracy, genetic analysis depends entirely on the complete genetic information of the proband and parents. For some at-risk fetuses without more than one causative variant found in the probands or when genetic analysis was not performed, this can hinder a precise prenatal diagnosis by genetic analysis alone. Presently, there were 12 fetuses without more than one causative variant and 4 fetuses whose genetic analysis were not performed. For these 16 fetuses, prenatal diagnosis was made depending on biochemical analysis alone. In a condition where the metabolite results were inconsistent, like the 5 fetuses in our study (Additional file 2), previous data indicates a preference for the more sensitive and specific biomarkers in the prenatal diagnosis [29]. Following this rule, among the 5 fetuses in the present study, 4 were diagnosed as the cblC defect. The one fetus diagnosed as unaffected showed a normal phenotype at the postnatal follow-up. Therefore, in this situation in which the genetic analysis alone did not permit a precise prenatal diagnosis, biochemical analysis for the supernatant of AF would provide fast and reliable results using a small amount of AF sample. The data could help families in making decisions concerning the pregnancies. This advantage was also observed in our previous reports on prenatal metabolite analysis in methylmalonic acidemia and glutaricacidemia-I [14, 30]. More importantly, the positive and negative predictive values for each metabolite in solo and in combination revealed that the combination of metabolites presented a greater reliability. Therefore, despite the accuracy of genetic analysis for prenatal diagnosis of cblC defect, the combination biochemical analysis of Hcy, C3, C3/C2, MMA, and MCA in AF appears to be 
valuable in the prenatal diagnosis of families for whom genetic results are not available.

\section{Conclusions}

The Hcy characteristic metabolite appears to be a sensitive biomarker for the prenatal diagnosis of cblC defect. The combination of the Hcy assay with acylcarnitine and organic acid analysis offers a fast, sensitive, and reliable prenatal diagnostic biochemical approach, which could overcome the challenge of the lack of genetic data for families at-risk of cblC defect fetuses.

\begin{abstract}
Abbreviations
cblC defect: Combined methylmalonic acidemia and homocystinuria, cobalamin C type; AF: Amniotic fluid; LC-MS/MS: Liquid chromatography tandem mass spectrometry; GC-MS: Gas chromatography mass spectrometry; Hcy: Homocysteine; MMA: Methylmalonic acid; C3: Propionylcarnitine; C2: Acetylcarnitine; MCA: Methylcitric acid.
\end{abstract}

\section{Supplementary Information}

The online version contains supplementary material available at https://doi. org/10.1186/s13023-021-01762-z.

Additional file 1. MMACHC gene variants for 232 probands and 56 affected fetuses. Description of data: The cDNA change, amino acid change, exon, effect and frequency of different MMACHC gene variants identified from 232 probands and 56 affected fetuses with cblC defect.

Additional file 2. Prenatal diagnostic results of 16 fetuses with inconclusive genetic results. Description of data: The metabolites' results and inconclusive genetic results of 16 fetuses.

\section{Acknowledgements}

Not applicable.

\section{Authors' contributions}

LH was responsible for the conception of the idea, acquisition of clinical data and supervision of the project. TC analyzed the initial clinical data and drafted the manuscript. LL and BX were major contributors in editing this manuscript. $\mathrm{HZ}$ and LW were responsible for the sample collection. TC, FX and ZG performed the measurements. All authors read and approved the final manuscript.

\section{Funding}

The National Key Research and Development Program of China (No.2016YFC0901505); Shanghai Municipal Health Commission (No. 202040448); The National Natural Science Foundation of China (No. 81600701).

\section{Availability of data and materials}

The datasets supporting the conclusions of this article are included within the article and its additional files.

\section{Declarations}

Ethics approval and consent to participate

This study was approved by the Ethics Committee of Xinhua Hospital (approval number XHEC-D-2020-131), and was conducted in agreement with the Declaration of Helsinki Principles.

\section{Consent for publication}

Not applicable.

\section{Competing interests}

The authors declare that they have no competing interests.

\section{Author details}

${ }^{1}$ Department of Pediatric Endocrinology and Genetic, Xinhua Hospital, Shanghai Institute for Pediatric Research, Shanghai Jiao Tong University School of Medicine, 1665 Kongjiang Road, Yangpu District, Shanghai 200092, China.

${ }^{2}$ Center for Prenatal Diagnosis, Xinhua Hospital, Shanghai Jiao Tong University School of Medicine, Shanghai 200092, China.

Received: 10 November 2020 Accepted: 25 February 2021

Published online: 10 March 2021

\section{References}

1. Nogueira C, Marcao A, Rocha H, Sousa C, Fonseca H, Valongo C, et al. Molecular picture of cobalamin C/D defects before and after newborn screening era. J Med Screen. 2017;24:6-11.

2. Weisfeld-Adams JD, Morrissey MA, Kirmse BM, Salveson BR, Wasserstein MP, McGuire PJ, et al. Newborn screening and early biochemical followup in combined methylmalonic aciduria and homocystinuria, cblC type, and utility of methionine as a secondary screening analyte. Mol Genet Metab. 2010;99:116-23.

3. Guo K, Zhou X, Chen X, Wu Y, Liu C, Kong Q. Expanded newborn screening for inborn errors of metabolism and genetic characteristics in a Chinese population. Front Genet. 2018;9:122.

4. Han B, Cao Z, Tian L, Zou H, Yang L, Zhu W, et al. Clinical presentation, gene analysis and outcomes in young patients with early-treated combined methylmalonic acidemia and homocysteinemia (cblC type) in Shandong province, China. Brain Dev. 2016;38:491-7.

5. Zhou W, Li H, Wang C, Wang X, Gu M. Newborn screening for methylmalonic acidemia in a Chinese population: molecular genetic confirmation and genotype phenotype correlations. Front Genet. 2018;9:726.

6. Hu S, Mei S, Liu N, Kong X. Molecular genetic characterization of cblC defects in 126 pedigrees and prenatal genetic diagnosis of pedigrees with combined methylmalonic aciduria and homocystinuria. BMC Med Genet. 2018;19:154.

7. Pollini L, Tolve M, Nardecchia F, Galosi S, Carducci C, di Carlo E, et al. Multiple sclerosis and intracellular cobalamin defect (MMACHC/PRDX1) comorbidity in a young male. Mol Genet Metab Rep. 2020;22:100560.

8. Martinelli D, Deodato F, Dionisi-Vici C. Cobalamin C defect: natural history, pathophysiology, and treatment. J Inherit Metab Dis. 2011;34:127-35.

9. Zhou X, Cui Y, Han J. Methylmalonic acidemia: current status and research priorities. Intractable Rare Dis Res. 2018;7:73-8.

10. Fischer S, Huemer M, Baumgartner M, Deodato F, Ballhausen D, Boneh A, et al. Clinical presentation and outcome in a series of 88 patients with the cblC defect. J Inherit Metab Dis. 2014;37:831-40.

11. Pastore A, Martinelli D, Piemonte F, Tozzi G, Boenzi S, Di Giovamberardino $\mathrm{G}$, et al. Glutathione metabolism in cobalamin deficiency type C (cblC). J Inherit Metab Dis. 2014;37:125-9.

12. Morel CF, Watkins D, Scott P, Rinaldo P, Rosenblatt DS. Prenatal diagnosis for methylmalonic acidemia and inborn errors of vitamin B12 metabolism and transport. Mol Genet Metab. 2005;86:160-71.

13. Zhang C, Wang X, Hao S, Zhang Q, Zheng L, Zhou B, et al. Mutation analysis, treatment and prenatal diagnosis of Chinese cases of methylmalonic acidemia. Sci Rep. 2020;10:12509.

14. Ji X, Wang H, Ye J, Qiu W, Zhang H, Liang L, et al. Prenatal diagnosis of methylmalonic aciduria from amniotic fluid using genetic and biochemical approaches. Prenat Diagn. 2019;39:993-7.

15. Huemer M, Diodato D, Schwahn B, Schiff M, Bandeira A, Benoist JF, et al. Guidelines for diagnosis and management of the cobalamin-related remethylation disorders cblC, cbID, cblE, cblF, cblG, cblJ and MTHFR deficiency. J Inherit Metab Dis. 2017;40:21-48.

16. Jiang Y, Mistretta B, Elsea S, Sun Q. Simultaneous determination of plasma total homocysteine and methionine by liquid chromatography-tandem mass spectrometry. Clin Chim Acta. 2017;464:93-7.

17. Hasegawa Y, Iga M, Kimura M, Shigematsu Y, Yamaguchi S. Prenatal diagnosis for organic acid disorders using two mass spectrometric methods, gas chromatography mass spectrometry and tandem mass spectrometry. J Chromatogr B Anal Technol Biomed Life Sci. 2005;823:13-7. 
18. Zong Y, Liu N, Zhao Z, Kong X. Prenatal diagnosis using genetic sequencing and identification of a novel mutation in MMACHC. BMC Med Genet. 2015;16:48.

19. Wang $X$, Yang Y, Li X, Li C, Wang C. Distinct clinical, neuroimaging and genetic profiles of late-onset cobalamin C defects (cb1C): a report of 16 Chinese cases. Orphanet J Rare Dis. 2019;14:109.

20. Carrillo-Carrasco N, Chandler RJ, Venditti CP. Combined methylmalonic acidemia and homocystinuria, cblC type. I. Clinical presentations, diagnosis and management. J Inherit Metab Dis. 2012;35:91-102.

21. Zhang Y, Yang YL, Hasegawa Y, Yamaguchi S, Shi CY, Song JQ, et al. Prenatal diagnosis of methylmalonic aciduria by analysis of organic acids and total homocysteine in amniotic fluid. Chin Med J (Engl). 2008;121:216-9.

22. Merinero B, Perez-Cerda C, Garcia MJ, Chadefaux-Vekemans B, Kamoun $P$, Tonetti $C$, et al. Reliability of biochemical parameters used in prenatal diagnosis of combined methylmalonic aciduria and homocystinuria. Prenat Diagn. 1998;18:947-52.

23. Sobolevsky T, Ahrens B. Measurement of urinary cobalt as its complex with 2-(5-chloro-2-pyridylazo)-5-diethylaminophenol by liquid chromatography-tandem mass spectrometry for the purpose of anti-doping control. Drug Test Anal. 2021. https://doi.org/10.1002/dta.3004.

24. Kerkhof P, Peace RA, Macfarlane PW. Sex- and age-related reference values in cardiology, with annotations and guidelines for interpretation. Adv Exp Med Biol. 2018;1065:677-706.

25. Liu Y, Ko EY, Wong KK, Chen X, Cheung WC, Law TS, et al. Endometrial microbiota in infertile women with and without chronic endometritis as diagnosed using a quantitative and reference range-based method. Fertil Steril. 2019;112:707-17.

26. McHugh D, Cameron CA, Abdenur JE, Abdulrahman M, Adair O, Al NS, et al. Clinical validation of cutoff target ranges in newborn screening of metabolic disorders by tandem mass spectrometry: a worldwide collaborative project. Genet Med. 2011;13:230-54

27. Gupta N, Kabra M, Haberle J. Mutation analysis of Indian patients with urea cycle defects. Indian Pediatr. 2012;49:585-6.

28. Nogueira C, Aiello C, Cerone R, Martins E, Caruso U, Moroni I, et al Spectrum of MMACHC mutations in Italian and Portuguese patients with combined methylmalonic aciduria and homocystinuria, cblC type. Mol Genet Metab. 2008:93:475-80.

29. Reitsma JB, Glas AS, Rutjes AW, Scholten RJ, Bossuyt PM, Zwinderman AH. Bivariate analysis of sensitivity and specificity produces informative summary measures in diagnostic reviews. J Clin Epidemiol. 2005;58:982-90.

30. Xiao B, Qiu W, Ye J, Zhang H, Zhu H, Wang L, et al. Prenatal diagnosis of glutaric acidemia I based on amniotic fluid samples in 42 families using genetic and biochemical approaches. Front Genet. 2020;11:496.

\section{Publisher's Note}

Springer Nature remains neutral with regard to jurisdictional claims in published maps and institutional affiliations.
Ready to submit your research? Choose BMC and benefit from:

- fast, convenient online submission

- thorough peer review by experienced researchers in your field

- rapid publication on acceptance

- support for research data, including large and complex data types

- gold Open Access which fosters wider collaboration and increased citations

- maximum visibility for your research: over $100 \mathrm{M}$ website views per year

At BMC, research is always in progress.

Learn more biomedcentral.com/submissions 